\title{
Curability of cancer by radiotherapy and chemotherapy, including in neuraxial neoplasms
}

\author{
Rakesh Jalali, Anusheel M unshi, Brijesh Arora ${ }^{1}$ \\ Departments of Radiation Oncology and ${ }^{1}$ Medical Oncology (Division of Pediatric Oncology), Tata Memorial Hospital, Mumbai, \\ India
}

\author{
Address for correspondence: \\ Dr. Rakesh J alali, \\ Radiation Oncology, \\ 125, Tata M emorial Hospital, \\ Parel, Mumbai - 400 012, India. \\ E-mail: rjalali@ tmc.gov.in
}

DOI: $10.4103 / 0028-3886.48794$

\begin{abstract}
In the 0 ctober of 1996, Lance Armstrong, celebrated cyclist and one of the greatest athletes the world has ever seen, at the age of 24 , was diagnosed with metastatic testicular cancer with disease having already spread to his abdomen, lungs and brain. Lance underwent four cycles of chemotherapy, actually the pretty standard one, pioneered at the Indiana U niversity and not only did he get completely cured of his cancer, he remains extremely well till date, 12 years later. He sure did have a few adverse effects during those cycles of chemotherapy in the form of nausea, vomiting, weakness and fall in blood count but he knew and experienced them only for a short transient time and emerged triumphant and strong. In fact, he went on to win six awe-inspiring and incredible successive Tours de France victories from 1999-2005, one of the most grueling sporting events testing the endurance of the very fittest. A fter his retirement, he has been so inspired that he has completely devoted himself to educate people about the common myths about cancer, and promised to raise awareness and generate money for furthering research into surgery, radiation therapy and chemotherapy for cancer through his foundation. He says "I am indebted to the doctors, nurses and medicine and would want to pay them back for all their energy and caring." In his successful journey of overcoming cancer, he captures the essence of its treatment so well by declaring "Pain is temporary, it may last a minute, or an hour, or a day, or a year, but eventually, it will subside and something else will take its place. If I quit, however, it will last forever".
\end{abstract}

Key words: C ancer, neuroaxial neoplasms, radiotherapy, chemotherapy

\section{Introduction}

The above case, by the way, is not exceptional; merely a reflection of millions of people having been cured of and survived cancer. Testicular cancer is the commonest cancer in the age group of 15 to 35 years of age $(8000$ new cases are diagnosed annually in the US and 48000 worldwide) with the cornerstone of management being chemotherapy and associated with remarkable cure rates of $70-100 \%$, even in advanced stages. ${ }^{[1]}$

Importantly, patients of course have to take the treatment (which fortunately a vast majority do) with an aim of 'cure' and cure they do achieve indeed. Readers of the recent article with an astonishingly nihilistic theme such an enormous evidence of cancer cures in the current era, may be led to believe that treatments of cancer are palliative with no chances of real cures. ${ }^{[2]}$ It would deprive these thousands of patients and indeed several million of cancer sufferers overall to continue suffering (and eventual death, often painful for there is no worse death than death from cancer) denying them the right and dignity to live as rightful individuals in the society. ${ }^{[3]}$ The above quoted editorial is seriously caught up in the time warp of the '50s and '60s, where such arguments could be justified to some extent. It suffers under the weight of antique anecdotes, rhyming quotes and poetic jargons negating the use of radiotherapy and 
chemotherapy, or advocating it for palliation at best (especially in neuraxial tumors) and pales miserably in front of the hard scientific evidence supporting its use for survival benefit in malignant neoplasms and neuraxial tumors. Cancer has already become a serious public health hazard where all individuals and medical communities have to come together and pledge support to prevent, treat, cure and look after cancer patients and survivors in the best possible way as we can. This is a tall order and mandates tremendous commitment and hope for the medical professionals and patients (and their caregivers) alike and calls for support throughout and certainly not by camouflaged half-truths, archaic anecdotes and ill-timed commentaries. As oncologists, it is our duty to apprise the reader of the truth, with hard evidence and leave the reader the choice of what to believe. As oncologists, we remain humble about the achievements so far and remain cognizant of the fact that there is still so much to learn and so many frontiers to conquer. We also are acutely aware of some of the adverse effects of the disease and therapy, which a cured survivor may face and no doubt view them with big neon lights for ourselves and our patients. We remain deeply committed to minimize these events and fortunately we have achieved this to some extent (see below). It is also true that a percentage of patients may not be cured and will still need treatment and support to palliate (palliation is not a bad word as sometimes perceived; it is as important as cure). But foremost as clinicians and human beings, who have to deal with the daily sufferings of a patient, a child in the clinic, we have to participate in the patients' journey with skill, knowledge, beliefs, humility and compassion.

\section{Cancer Survival and Cures}

Cure in cancer, or for that matter in any chronic disease (diabetes, hypertension, arthritis and a host of other such illnesses) is difficult to define. It could be argued that by modern medicine, we are palliating and not really 'curing' diabetes and hypertension as well and yet you will hardly ever see such writings and opinions as reserved for 'cancer'. In fact, if at all, we have a degree of measuring 'cure' in cancer in comparison to the above mentioned chronic disease. Evidence of an absence of disease clinically, biochemically, radiologically and now even genetically is generally deemed as cure. Indeed, in several leukemias, we are now able to measure cures by demonstrating reversal of chromosomal anomalies characterizing the particular type of cancer. Such pathbreaking success stories of chemotherapy (in acute leukemias, commonest form of cancer in children with $>70-80 \%$ cures) and biological therapies in chronic leukemias in adults are one of the finest examples of achieving 'cures' by any definition and a fitting reply to the 'only palliation' paradigm.
It is also true that a majority of cancers recur in the first two to three years of diagnosis and hence a five-year survival has traditionally been equated with cures. We have understood that this may not be the case in every single case and in some cancers such as breast cancer, late recurrences are known. Yet, five-year survival as a marker for cure holds true for more than $80-90 \%$ of cancers and can be used as a reasonable measure. In developed countries like the United States, five-year relative survival rates for all cancers combined have increased steadily, from $50 \%$ in $1975-79$ to $66 \%$ in $1996-$ 2002 among adults, and from $61-79 \%$ among children. ${ }^{[4]}$ For the skeptics, we now have even 10-year survival rates available, which reveal survivorship of $59 \%$ in adults and $75 \%$ in children. ${ }^{[5]}$

These are indeed very encouraging figures by any standards and by any yardstick. Such figures have been possible by increasing awareness, early detection and modern therapies (surgery, radiation, chemotherapy, biological therapy). In developing countries such as ours, where we have huge challenges of effective screening policies, late detection of cancer, lack of widespread treatment facilities and knowledge, fiveyear survival rates are between $30-40 \%{ }^{[6]}$ At a global level, out of the 12 million cases of cancer diagnosed annually, 5.4 million are from the economically developed countries and 6.7 from the developing countries. Importantly, almost half of them can be expected to be cured (not palliated alone) with modern medicine.

\section{Radiotherapy}

Radiation therapy has completed more than 100 years of effective use for management of malignant tumors. During this time, it has been used for nearly all known cancers and has saved millions of lives all over the globe. For its effect on malignant tumors, radiation therapy relies on a therapeutic differential between malignant and normal cells. On a cellular basis, this in turn is based on differential proliferative potential, varying cell repair kinetics and preferential damage to DNA of malignant cells. ${ }^{[7]} \mathrm{A}$ few examples:

1. Radiation can be used as curative intent treatment in virtually every stage of uterine cervical cancer. With current treatment protocols, radiation therapy can achieve long-term cure rates of $85-90 \%$ in Stage I, 60-80\% in Stage II, 30-50\% in Stage III, and $10-20 \%$ even in Stage IVA. ${ }^{[8]}$ Imagine a lady with cervical cancer (the most common cancer in India with 70,000 cases diagnosed every year in India alone and 493,000 worldwide) not offered treatment with radiotherapy, on the basis of some of the 'palliative mode' thinkers? Would it not be unethical? 
2. Radiation is used as a curative treatment in a broad spectrum of head and neck cancers. While in early lesions it competes with surgery as the local treatment of choice, both modalities are often used together whenever possible as a combined modality approach in locally advanced cancers. In case of vocal cord lesions, radiotherapy provides a unique opportunity for organ preservation with a consequent improved quality of life (QOL) ${ }^{\left[{ }^{[9]}\right.}$ Cure rates in node-negative T1/T2 lesions treated with radical radiation incorporating radiotherapy have been between $80-95 \%$. In locally advanced lesions, radiation is a very important part of combined modality treatment. Conformal radiotherapy, especially intensity modulated radiotherapy (IMRT) has enabled the delivery of higher doses of radiation to the target volumes while sparing healthy normal tissues. ${ }^{[10]}$

3. Radical radiotherapy is a viable alternative to radical prostatectomy in the first-line management of early stage prostate cancer. The classical 10-year cause-specific survival rates with radical radiation for T1, T2, T3 and T4 tumors are 79\%, 66\%, 55\%, and $22 \%$ respectively. ${ }^{[11]}$ The use of IMRT has enabled the delivery of high doses within the pelvis and has improved the results of radical radiation to equal to that of surgery. In locally advanced prostate cancer, the use of radiation along with hormonal treatment is the standard of care with survivals close to $80 \%$ at five years. ${ }^{[12]}$

\section{Chemotherapy}

Since the advent of chemotherapy in the treatment of cancer more than 50 years ago, prognosis of a majority of adult and pediatric solid tumors has improved considerably. Furthermore, strides taken in chemotherapy in the treatment of hematological cancers such as leukemias, lymphoma, myeloma and myelodysplasia are some of the greatest success stories of modern medicine. Analogous to radiotherapy, chemotherapy eradicates or kills all cancer cells in the body by exploiting the kinetic proliferative differences between the cancer cell and the normal host cell. Cells are targeted in various phases of the cycle achieving a discernible differential response at the cellular level between normal and malignant cells. Some of the key examples are:

1. Chemotherapy in germ cell tumors achieves high cure rates to the tune of $90-100 \%$ in the early stage and $60-70 \%$ in advanced stage. ${ }^{[13,14]}$

2. Lymphomas, like germ cell tumors, are extremely radiosensitive and chemosensitive tumors. Radiotherapy has played a pivotal role in the evolution of curative treatment of lymphomas, especially Hodgkin's disease. The current management of early stage Hodgkin's has evolved from the use of curative radiation alone to combined modality treatments incorporating highly effective combination chemotherapy and involved field radiotherapy. Long-term cure rates of $80-95 \%$ have been achieved in Hodgkin's disease. ${ }^{[15,16]}$

\section{Pediatric cancers}

Survival rates for children 0 to 14 years of age have improved dramatically since the 1960s after the introduction of chemotherapy when the overall five-year survival rate after a cancer diagnosis was estimated at 28\%. ${ }^{[17]}$ Improvement in survival rates continues with current three-year survival rates exceeding $80 \%$ and five-year survival rates exceeding $75 \%$ for children and adolescents with cancer. The increase in survival for children younger than 15 years has been most dramatic for acute lymphoblastic leukaemia (ALL), a virtually incurable disease in the early '60s and for which fiveyear survival rates exceeded 80\% in the period from 1989 through 2001. Survival rates for childhood lymphomas have increased to nearly 80\% from 1989 through 2001, up from $20 \%$ to $25 \%$ in the early ' 60 s, and survival rates for Wilms' tumor increased from 33\% in 1960 to over 90\% today. ${ }^{[18]}$ Five-year survival rates at or above $70-90 \%$ have also been achieved for Hodgkin's disease, retinoblastoma, thyroid cancer, pediatric sarcomas, brain tumors, neuroblastomas, melanoma and Ewing's sarcoma. ${ }^{[19]}$ This has been made possible by effective utilization of chemotherapy in hematological malignancies and use of chemotherapy and radiotherapy in addition to functionpreserving surgery in solid tumors.

\section{Neuro-axial tumors}

We do want to talk a little bit about tumors arising from the central nervous system (CNS), a subject not unfamiliar with the readers. While CNS tumors may comprise about $1-2 \%$ of all cancers, they are interesting in the range of pathologies from benign to most malignant (glioblastoma, metastasis) affecting virtually all age groups from infants, children, adults and to very old making neurooncology fascinating. In children, they are the commonest solid cancers (second commonest overall after leukemias). CNS tumors also pose unique management issues mandating a close cooperation between various health professionals and also a major focus not only in survivorship but quality of survivorship as well. The management of the commonest neuro-axial tumors of childhood, medulloblastoma malignant, Grade IV tumor), in fact is a good example to demonstrate the efficacy of adjuvant treatments. It is well known to the neurosurgical and oncology community that almost all patients after a surgical resection (which is a very important part of management no doubt) if left alone, will recur and die with no survivors untreated or treated by surgery alone. 
It was the great Harvey Cushing who observed "In the course of our growing acquaintance with these baffling tumors, we suspected from their peculiar cytology that they might be susceptible to radiation and the first of the cases so treated both by the X-rays and radium was in December, 1919. Here at least was a new therapeutic recourse and we began with renewed encouragement to attack them with renewed vigor". A study from the '60s suggested a fiveyear survival of $35 \%$ and a 10 -year survival of $26 \%$ after radiation therapy following surgery. ${ }^{[20]}$ Since then, there have been rapid strides and evolution of techniques of radiotherapy, doses and incorporation of chemotherapy in the management paradigms. Recent trials have shown that craniospinal radiotherapy with precision techniques, together with chemotherapy, can achieve $>85 \%$ five-year overall survival in average risk disease and $50 \%$ survival even in high-risk (metastatic) disease. ${ }^{[21]}$ High cure rates of more than $70-90 \%$ have been achieved with a combination of radiation and chemotherapy in CNS germ cell tumors, another tumor affecting the entire neuraxis. ${ }^{[22]}$

The efficacy of radiotherapy has been equally proven in other tumors as well. Survival benefit of radiotherapy has been demonstrated in glial neoplasms, the most common primary tumors in adults. ${ }^{[23]}$ Also, the addition of chemotherapy (Temozolomide) has contributed significantly to improvement in overall survival in such patients, although failure to achieve high cures in malignant gliomas still remains one of the biggest challenges in neurooncology. ${ }^{[24]}$ In infants and young children ( $<$ five years) afflicted by brain tumors, chemotherapy helps in preserving cognition by preventing or delaying the usage of radiotherapy in more than two-thirds of cases.

\section{Organ preservation in malignant disorders}

Organ preservation is a unique concept that allows the patient to retain the organ with acceptable function and cosmetic outcome. This concept has gained steady acceptability for an increasing number of sites in the body. The principle of organ preservation involves a combination of radiotherapy and chemotherapy for control of disease and preservation of useful organ function, keeping surgery as salvage in few selected non-responders. ${ }^{[25]}$ This approach has been found especially useful in laryngeal, breast, anorectal and bladder malignancies with proven long-term efficacy both in terms of disease control and survival and organ preservation rates, leading to a better quality of life.

Multimodality therapy in bladder implies transurethral resection of the bladder tumor combined with chemoradiation therapy. This approach yields a five-year survival rate with bladder conservation of $36-48 \%$ with an overall survival rate of $48-63 \% .^{[26]}$ Breast conservation in breast malignancies has been made possible by post lumpectomy adjuvant radiotherapy to the whole breast, thus offering excellent cosmetic outcome to females who wish to retain their breasts.

Secondary cancers after radiation and chemotherapy This issue of secondary cancers after radiotherapy and chemotherapy has been keenly debated and often exaggerated. First of all, there is no doubt that radiation and chemotherapeutic agents are potentially carcinogenic but we must look at the incidences of second cancers objectively. In the data analyzed from 2,056 five-year survivors of primary brain cancer in the surveillance, epidemiology, and end results (SEER) database between 1973 and 1998, 39 patients developed second neoplasms, an incidence of $<1.5 \%$. ${ }^{[27]}$ Half of the second neoplasms were meningiomas, which of course carry high cure rates after a surgical resection. In view of such low incidences, should we not offer radiation and chemotherapy to patients with CNS tumors? Treatment with chemotherapy and radiotherapy may lead to several point and segment chronic mutations. These mutations and genetic changes lead to second malignancies in these patients, years after they are cured of the original cancer. On the other hand, if no treatment with these modalities is offered to these patients, there will be progression/ recurrence of the disease and will lead to severe morbidity in these patients. Thus, standard protocol is to weight the benefits of chemotherapy and radiotherapy in preventing recurrence/progression of the disease and have an 'acceptable risk' of second malignancy or other long-term toxicities. Probability of second malignancy depends not only upon the treatment modalities used, but also on treatment and patient-related factors. Larger radiation field size, early age of treatment, associated use of carcinogenic chemotherapeutic agents, patient's personal habits, like continuation of smoking tobacco or tobacco chewing and patient susceptibility due to genetic disorders (e.g. xeroderma pigmentosa) are associated with higher probability of second malignancy. Especially the aerodigestive system which is exposed to the tobacco or environmental carcinogens is at continuous risk of multiple cancers at different sites called 'field cancerization'. Patients exposed to carcinogens like tobacco even after a cancer have a higher probability of developing multiple cancers irrespective of the treatment modality.

'Multi-centric tumor' appearing at various sites and 'multiple tumors at different sites' are difficult to distinguish and all these tumors are estimated in second malignancy giving a false over-estimation of second malignancies. Thus, before interpretation of the effect of treatment modalities in developing second cancers, patients with high risk of second cancer (e.g. genetic susceptibility, continuous exposure to carcinogens) unrelated with the treatment modality should be excluded 
to eliminate the false high incidence of second cancers in cancer survivors. Unfortunately, majority of the second cancer data have not taken these factors into account during estimation of second cancer incidence. Despite above mentioned problems in interpretation of 'second malignancy', surveillance, epidemiology, and end results has compiled 27-year data from 1973 to 2000 regarding the risk of second malignancy in cancer survivors. Based on the nine original cancer registries, the SEER Program provided data on more than two million cancer survivors (including nearly 390,000 patients surviving at least 10 years and 76,000 patients surviving 20 or more years), yielding close to 11 million person-years at risk over the follow-up period from 1973 to 2000. Overall, the 25-year cumulative incidence of developing a second cancer was only $3.5 \%(95 \% \mathrm{CI}=3.0 \%-4.1 \%){ }^{[28]}$

\section{Acute toxicity of surgery, radiotherapy and chemotherapy}

Each cancer-directed therapy has some side-effects. While in surgically treated cancers, there is significant structural and functional local morbidity including loss of organ function, cosmetic defects or rarely death; most patients receiving curative or palliative intent radiotherapy or chemotherapy would experience some acute systemic complication such as skin redness, cytopaenias, infections, mucositis, nausea, vomiting, diarrhea, hair loss etc. It is true that rapidly dividing normal tissues such as bone marrow, skin and hair follicles are subject to effects of anti-proliferative agents such as chemotherapy. Radiotherapy on the other hand is a localized treatment and hence affects only those parts in the field of treatment. Because of the differential between these tissues and malignant tissue, these side-effects are temporary and are well managed with current modalities of specific antiemetics, growth factors, transfusions, ports, antibiotics and other medications. The oncology community has been very much conscious of these effects and organizes several programs such as support groups, counseling sessions etc, which have been very successful in alleviating the anxieties. All of these are transient effects, but are often exaggerated by non-oncology communities.

\section{Chronic toxicities in cancer survivors}

It is well recognized that cancer survivors may exhibit late sequelae in the form of cognitive dysfunction, motor deficit, visual impairment, hormonal dysfunction, psychological-emotional problems, growth deficits, stroke (primarily in CNS tumors), xerostomia (in head and neck cancers), cardiac morbidity (in thoracic malignancies), rectal/bladder toxicities (in pelvic tumors) and second malignant neoplasms. These effects are a due to a combination of their tumors, surgery, radiation/chemotherapy and other environmental factors and it has always been a challenge to measure the contribution of each. A lot of effort has been devoted to prevent, minimize and treat these effects with support from the physicians, support groups and experts in these fields. Separate task forces and committees have been formed to address these issues. In view of the tremendous refinement in the techniques of radiotherapy (conformal, IMRT, stereotactic radiotherapy), we are already witnessing a reduction in these morbidities to a considerable extent. For instance, in breast cancer, for women irradiated during 1973-82, 1983-92 and 19932001, the cardiac mortality ratio (left versus right tumor laterality) was $1.42(1.11-1.82), 1.27(0.99-1 \cdot 63)$ and none respectively, indicating a clear reduction in cardiac mortality with newer technology. ${ }^{[29]}$ Survivors of head and neck cancers who received conformal radiotherapy had a 2.01-fold higher probability of reporting good global QOL and a 2.70-fold lower probability of reporting a high level of xerostomia than survivors who received conventional radiotherapy. ${ }^{[30]}$ Similar decrease has been reported in pelvic tumors with respect to bowel and bladder toxicities with use of conformal techniques. ${ }^{[31]}$

In CNS tumors, one of the major issues has been neuropsychological impairment, which has been reported to be $20 \%$ to $60 \%$ of the long-term survivors in earlier series. It has been associated with various patient, disease and treatment-related parameters such as tumor in the supratentorial region, tumor involving hypothalamus, hydrocephalus, shunt, uncorrected hormone deficiency, epilepsy, use of chemotherapy or radiotherapy. It is again difficult to ascertain from these reports the exact contribution of each of these factors and how they interact with each other. It is fair to assume of course that radiotherapy is at least partly responsible although its exact role is yet to be quantified. Recent reports including our data have revealed that as many as two-thirds of children with brain tumors have impaired cognition, even before starting radiotherapy suggesting that factors like tumor, hydrocephalus, surgery etc to have a role to play as well. ${ }^{[32]}$ Conformal radiotherapy techniques have also preserved the IQ levels and prevented any further decline, even in children less than three years of age. ${ }^{[33,34]}$ Reduction of craniospinal irradiation (CSI) doses for primitive neuroectrodermal tumours (PNET's), avoidance of radiation to the very young and use of optimal doses and highly conformal radiotherapy techniques are some of the steps taken in the last few years and have already shown promising results. ${ }^{[23]}$ Similarly, hypopituitarism occurs due to a combination of factors including tumor per se, surgery and radiation therapy also contributes to some extent. More than half of patients with low-grade brain tumors before radiotherapy have been shown to have hormone deficiency in at least one axis, with maximum deficiency seen in sellar tumors and only $3 / 57$ patients needed an additional hormone after treatment with modern stereotactic conformal 
radiotherapy. ${ }^{[35]}$ Radiation-induced optic neuropathy is virtually unknown now in clinical practice with the doses, fractionation and techniques employed. Risk of cerebrovascular accident and radionecrosis are seen in approximately $1 \%$ of patients. In summary, while cancer survivors are at a risk of developing some late events, these are likely to be due to a combination of factors including tumors per se. Judicious use of modern modalities of surgery, radiotherapy and chemotherapy minimizes several of these sequelae.

\section{Opinions Vis-à-vis Evidence in the Contemporary Era}

Science (and medicine) demands evidence and we are fortunate that we do indeed have reasonable evidence to counter the opinions in the editorial, which has prompted this rebuttal. A careful analysis of the quoted aphorisms ranging from Shakespeare $\left(16^{\text {th }}-17^{\text {th }}\right.$ century), Voltaire $\left(17^{\text {th }}-18^{\text {th }}\right.$ century) and others, no doubt literary giants and celebrated philosophers, gives us a glimpse of the topicality and tone of the article. A scrutiny of specific examples quoted is as follows:

1. Celebrated novelist Solzhenitsyn's work Cancer ward was actually published in 1968 (the year was not mentioned in the editorial) and was based on his experiences in the oppressed erstwhile Soviet regimen. It is not surprising that the doctors in that era did not fully comprehend the usage of radiation and chemotherapy (only a few drugs were available). 'Harsh X-rays' of those times (orthovoltage, 120-250 KV) have been completely abandoned for the last 20-30 years in clinical practice throughout the world; modern radiation beams (while still electromagnetic in property) have much higher energy (megavoltage, 6-18 MV) and vastly sharper beams with the ability to literally sculpt the doses around all complex tumor shapes. Modern radiotherapy with the help of imaging and conformal devices adapts its shape of delivery to the shape of potential tumor volumes such that a high dose hitherto not possible to deliver, can be accomplished with already clinically demonstrable improved therapeutic ratios of high cures and least morbidity. ${ }^{[36,37]}$

2. Glemser's work was published in 1969 (year was not mentioned) based on cross-sectional interviews of oncologists around the world. That chemotherapy was dubbed as 'absolute farce' is really not a surprise, for there were only a few drugs available that time. Everyone knows that the real breakthrough in chemotherapy came in the '70s and '80s when drugs for leukemias, lymphomas, germ cell tumors, breast cancer etc were employed with a lot, lot more success. It would be interesting to perform a survey in the modern era and compare notes with Glemser (who by the way also dismissed surgery for cancer as 'avoidable').

3. The comment on 'chemotherapy' in the Youmans Neurological Surgery, $5^{\text {th }}$ edition in 2004 that it "can benefit to a modest extent" is true as traditional chemotherapeutic agents do not penetrate the blood brain barrier. Yet, efficacy of chemotherapeutic agents in the evolving success in childhood PNET's and germ cell tumors and encouraging survival gains even in malignant gliomas (as illustrated in earlier sections) in the last five years or so cannot be ignored and is very likely to find a mention in their next edition.

4. Dudley's comment in British Journal of Medicine in 1978 about not undergoing an abdominoperineal resection for a rectal cancer is well known in oncology circles. It is also quite true that sphincter-preserving surgery is possible in more than $50-70 \%$ of patients these days (with chemoradiation) and reflects the change in practice over time. There are also individuals who would prefer to live (and contribute positively to the society) even with a colostomy, if need be, than not have treatment and die of cancer.

5. The observation by Current Medical Diagnostics 2008 that " $76 \%$ cancers are diagnosed in persons more than 75 years of age" is probably correct. The figures pertain to the Western world statistics and the role of radiation/chemotherapy as a contributing factor is very minor (well illustrated above in the section on second cancers). An overwhelming majority of cancers in this age group are due to genetic wear/tear and lifestylerelated. That so many cancers are seen at such an advanced age actually is not a worry for employing radiation and chemotherapy, at least from their potential risk of causing a second cancer in this ageing population.

Finally, our MOTTO has always been to weigh the advantages and risks posed by cancer and its treatment. While we can hope that a half to two-thirds of our patients can be cured in the long term, we remain deeply cognizant of the effects of treatment, survivorship issues, geographical disparities in treatment facilities around the globe, increasing awareness of prevention and early detection (education and screening), need for more effort towards research and cures for still many cancers, effective palliation and terminal care, etc. We of course also continue to have a discourse with fellow professionals, researchers, patients, caregivers, charity groups, governmental and non-governmental organizations. Issues such as these 
continue to light our fire of commitment and quest to conquer cancer and its barriers.

\section{References}

1. Matsuda T, Saika K. Comparison of time trends in testicular cancer incidence (1973-97) in East Asia, Europe and USA, from Cancer Incidence in Five Continents Vols IV-VIII. Jpn J Clin Oncol 2008;38:578-9.

2. Kothari M, Goel A. Integrity, immunity, reactivity, restorativity: Biolessons off brain abscess. Neurol India 2008;56:397-8.

3. Kothari M, Goel A. Anticytotics - Radiopalliation/chemopalliation and neuraxial neoplasms. Neurol India 2008;56:113-5.

4. Kamangar F, Dores GM, Anderson WF. Patterns of cancer incidence, mortality, and prevalence across five continents: Defining priorities to reduce cancer disparities in different geographic regions of the world. J Clin Oncol 2006;24:2137-50.

5. Parkin DM, Bray F, Ferlay J, Pisani P. Global cancer statistics, 2002. Ca Cancer J Clin 2005; 55:74-108

6. Sankaranarayanan R. Commentary: Cancer incidence among Asian Indians in India and abroad. Int J Epidemiol 2008;37:160-1.

7. Dunne-Daly CF. Principles of radiotherapy and radiobiology. Semin Oncol Nurs 1999;15:250-9.

8. Ota T, Takeshima N, Tabata T, Hasumi K, Takizawa K. Treatment of squamous cell carcinoma of the uterine cervix with radiation therapy alone: Long-term survival, late complications, and incidence of second cancers. Br J Cancer 2007;97:1058-62.

9. Mendenhall WM, Amdur RJ, Morris CG, Hinerman RW. T1-T2N0 squamous cell carcinoma of the glottic larynx treated with radiation therapy. J Clin Oncol 2001;19:4029-36.

10. Portelance L, Chao KS, Grigsby PW, Bennet H, Low D. Intensitymodulated radiation therapy (IMRT) reduces small bowel, rectum and bladder doses in patients with cervical cancer receiving pelvic and paraaortic irradiation. Int J Radiat Oncol Biol Phys 2001;51:261-6.

11. del Regato JA, Trailins AH, Pittman DD. Twenty years follow-up of patients with inoperable cancer of the prostate (stage C) treated by radiotherapy: Report of a national cooperative study. Int J Radiat Oncol Biol Phys 1993;26:197-201.

12. Bolla M, Collette L, Blank L, Warde P, Dubois JB, Mirimanoff RO, et al. Long-term results with immediate androgen suppression and external irradiation in patients with locally advanced prostate cancer (an EORTC study): A phase III randomised trial. Lancet 2002;360:103-6.

13. Fossa SD, Horwich A, Russell JM, Roberts JT, Cullen MH, Hodson NJ, et al. Optimal planning target volume for stage I testicular seminoma: A Medical Research Council randomized trial. J Clin Oncol $1999 ; 17: 1146-52$.

14. Classen J, Souchon R, Hehr T, Bamberg M. Radiotherapy for early stages testicular seminoma: Patterns of care study in Germany. Radiother Oncol 2002;63:179-86.

15. Girinsky T, Ghalibafian M. Radiotherapy of Hodgkin lymphoma: Indications, new fields, and techniques. Semin Radiat Oncol 2007;17:206-22.

16. Nogová L, Reineke T, Brillant C, Sieniawski M, Rüdiger T, Josting A, et al. Lymphocyte-predominant and classical Hodgkin's lymphoma: A comprehensive analysis from the German Hodgkin Study Group. J Clin Oncol 2008;26:434-9.

17. Pediatric Cancers International Encyclopedia of Public Health, 2008. p. $28-40$.

18. Sonn G, Shortliffe LM. Management of Wilms tumor: Current standard of care. Nat Clin Pract Urol 2008;5:551-60.

19. Ludwig JA. Ewing sarcoma: Historical perspectives, current stateof-the-art and opportunities for targeted therapy in the future. Curr
Opin Oncol 2008;20:412-8.

20. Aron BS. Twenty years' experience with radiation therapy of medulloblastoma. AJR Am J Roentgenol 1969;105:37-42.

21. Packer RJ. Progress and challenges in childhood brain tumors. J Neurooncol 2005;75:239-42.

22. Bamberg M, Kortmann RD, Calaminus G, Becker G, Meisner C, Harms D, et al. Radiation therapy for intracranial germinoma: Results of the German cooperative prospective trials MAKEI 83/86/89. J Clin Oncol 1999;17:2585-92.

23. Walker MD, Strike TA, Sheline GE. An analysis of dose-effect relationship in the radiotherapy of malignant gliomas. Int J Radiat Oncol Biol Phys 1979;5:1725-31

24. Stupp R, Mason WP, van den Brent MJ, Weller M, Fisher B, Taphoorn MJ, et al. Radiotherapy plus concomitant and adjuvant temozolomide for newly diagnosed glioblastoma. N Engl J Med 2005;352:987-96.

25. Tufano RP, Stafford EM. Organ preservation surgery for laryngeal cancer. Otolaryngol Clin North Am 2008;41:741-55.

26. Hosseini SY. Invasive bladder cancer: The role of bladder preserving therapy. Urol J 2005;2:1-7.

27. Peterson KM, Shao C, McCarter R, MacDonald TJ, Byrne J. An analysis of seer data of increasing risk of secondary malignant neoplasms among long-term survivors of childhood brain tumors. Pediatr Blood Cancer 2006;47:83-8.

28. Available from: http://seer.cancer.gov.

29. Darby SC, McGale P, Taylor CW, Peto R. Long-term mortality from heart disease and lung cancer after radiotherapy for early breast cancer: Prospective cohort study of about 300000 women in US SEER cancer registries. Lancet Oncol 2005;6:557-65.

30. Fang FM, Tsai WL, Chen HC, Hsu HC, Hsiung CY, Chien CY, et al. Intensity-modulated or conformal radiotherapy improves the quality of life of patients with nasopharyngeal carcinoma: Comparisons of four radiotherapy techniques. Cancer 2007;109:313-21.

31. Portelance L, Chao KS, Grigsby PW, Bennet H, Low D. Intensitymodulated radiation therapy (IMRT) reduces small bowel, rectum, and bladder doses in patients with cervical cancer receiving pelvic and paraaortic irradiation. Int J Radiat Oncol Biol Phys 2001;51:261-6.

32. Carpentieri SC, Waber DP, Pomeroy SL, Scott RM, Goumnerova LC, Kieran MW, et al. Neuropsychological functioning after surgery in children treated for brain tumor. Neurosurg 2003;52:1348-56.

33. Jalali R, Goswami S, Sarin R. Neuropsychological status in children and young adults with benign and low-grade brain tumors treated prospectively with focal stereotactic conformal radiotherapy. Int J Radiat Oncol Biol Phys 2006;66:S14-9.

34. Merchant TE, Mulhern RK, Krasin MJ, Kun LE, Williams T, Li C, et al. Preliminary results from a phase II trial of conformal radiation therapy and evaluation of radiation related CNS effects for pediatric patients with localised ependymoma. J Clin Oncol 2004;22:3156-62.

35. Dutta D, Shah N, Gupta T, Munshi A, Jalali R. Prospective analysis of endocrine function in children with residual/recurrent low-grade brain tumors treated with high-precision stereotactic conformal radiotherapy. J Clin Oncol 2008;26:2049.

36. Kam MK, Leung SF, Zee B, Chau RM, Suen JJ, Mo F, Lai M, et al. Prospective randomized study of intensity-modulated radiotherapy on salivary gland function in early-stage nasopharyngeal carcinoma patients. J Clin Oncol 2007;25:4873-9.

37. Zelefsky MJ, Levin EJ, Hunt M, Yamada Y, Shippy AM, Jackson A, et al. Incidence of late rectal and urinary toxicities after three-dimensional conformal radiotherapy and intensity-modulated radiotherapy for localized prostate cancer. Int J Radiat Oncol Biol Phys 2008;70:1124-9.

Accepted on 22-11-2009

Source of Support: Nil, Conflict of Interest: None declared. 